\title{
GOOD REDUCTION OF K3 SURFACES IN EQUICHARACTERISTIC $p$
}

\author{
BRUNO CHIARELLOTTO, CHRISTOPHER LAZDA, AND CHRISTIAN LIEDTKE
}

\begin{abstract}
We show that for smooth and proper varieties over local fields with no non-trivial vector fields, good reduction descends over purely inseparable extensions. We use this to extend the Néron-Ogg-Shafarevich criterion for K3 surfaces of [CLL17] to the equicharacteristic $p>0$ case.
\end{abstract}

\section{Contents}

Introduction 1

1. Review of $p$-adic cohomology in equicharacteristic $p>0 \quad 4$

2. Descending good reduction under separable extensions 8

3. Descending good reduction under purely inseparable extensions $\quad 10$

4. Main results 13

5. A counter-example 13

$\begin{array}{ll}\text { References } & 14\end{array}$

\section{INTRODUCTION}

Let $\mathcal{O}_{K}$ be an excellent Henselian DVR with perfect residue field $k$ and fraction field $K$. Let $K^{\mathrm{s}}$ be a separable closure of $K$ and let $G_{K}=\operatorname{Gal}\left(K^{\mathrm{s}} / K\right)$ be the corresponding absolute Galois group. Then, the residue field $\bar{k}$ of $K^{\mathrm{s}}$ is an algebraic closure of $k$ and we let $G_{k}$ denote the corresponding absolute Galois group. Let $X$ be a K3 surface over $K$.

Definition. A model for $X$ over $\mathcal{O}_{K}$ is a flat and proper morphism $\mathcal{X} \rightarrow \operatorname{Spec}\left(\mathcal{O}_{K}\right)$ of algebraic spaces together with an isomorphism $X \stackrel{\cong}{\longrightarrow} \mathcal{X}_{K}$.

We say that $X$ has good reduction if it admits a smooth model over $\mathcal{O}_{K}$. It follows from the smooth and proper base change theorem that if $X$ has good reduction, then the $G_{K}$-action on $H_{\text {ét }}^{2}\left(X_{K^{\mathrm{s}}}, \mathbb{Q}_{\ell}\right)$ is unramified for any prime $\ell \neq \operatorname{char}(k)$. In order to study the extent to which the converse of this is true, we consider the following (strong) notion of semistable reduction.

Assumption $(\star)$. A K3 surface $X$ over $K$ satisfies $(\star)$ if there exists a finite field extension $L / K$ and a model $\mathcal{X}$ for $X_{L}$ over $\mathcal{O}_{K}$ such that:

(1) the special fibre of $\mathcal{X}$ is a strict normal crossing divisor;

(2) the relative canonical divisor $\omega_{\mathcal{X} / \mathcal{O}_{L}}$ is trivial.

In honour of Kulikov's results [Kul77], such a model $\mathcal{X}$ is called a Kulikov model. In equicharacteristic zero, Assumption ( $\star$ ) is always satisfied [Kul77,PP81]. Also, if the residue characteristic $p$ is at least 5 , Assumption $(\star)$ is satisfied whenever $X$ has potential scheme theoretic semistable 
reduction [LM18, Mau14]. For example, this is satisfied if $X$ admits an ample invertible sheaf $\mathcal{L}$, whose self-intersection number satisfies $\mathcal{L}^{2}+4<p$. We refer to [LM18, §3] for further results and discussion.

Good reduction in characteristic zero. If $\operatorname{char}(K)=0$, then converse results, that is, NéronOgg-Shafarevich criteria for good reductions of K3 surfaces have been established in [CLL17,LM18, Mat15]. More precisely, the main result (Theorem 1.3) of [LM18] reads as follows.

Theorem 1 (Liedtke-Matsumoto). Assume that $\operatorname{char}(K)=0$, let $X$ be a K3 surface over $K$, satisfying Assumption $(\star)$, and let $\ell$ be a prime $\neq \operatorname{char}(k)$. Consider the following three statements:

(1) the $G_{K}$-action on $H_{\text {êt }}^{2}\left(X_{K^{\mathrm{s}}}, \mathbb{Q}_{\ell}\right)$ is unramified;

(2) $X$ has good reduction over a finite and unramified extension $L / K$;

(3) $X$ admits a model $\mathcal{X}$ that is projective over $\mathcal{O}_{K}$ and whose special fibre is a K3 surface with at worst $R D P$ singularities.

Then, we have $(1) \Leftrightarrow(2) \Rightarrow(3)$.

Remark. There exist examples of K3 surfaces over $\mathbb{Q}_{p}$ with $p \geq 5$ that only admit good reduction over a non-trivial unramified extension, see [LM18, Theorem 1.6]. We will show how to modify these to produce similar examples over Laurent series fields $\mathbb{F}_{p}((t))$ in $\S 5$ below.

This begs the question of whether there exists a criterion that sees good reduction 'on the nose'. This was the question investigated in [CLL17] and to explain the answer, we note that any K3 surface $X / K$ satisfying (1), (2) and (3) in Theorem 1 has a 'canonical reduction' $Y$, which is a K3 surface over $k$. Concretely, this is given as the minimal resolution of singularities of the special fibre of any RDP model $\mathcal{X}$ as in (3). We note that $Y$ is unique up to canonical isomorphism and does not depend on the choice of model. The main result (Theorem 1.6) of [CLL17] is then the following.

Theorem 2 (Chiarellotto-Lazda-Liedtke). Assume that $\operatorname{char}(K)=0$, let $X$ be a K3 surface over $K$, satisfying Assumption $(\star)$ and let $\ell$ be a prime $\neq \operatorname{char}(k)$. Then, then following are equivalent:

(1) $X$ has good reduction over $K$;

(2) the $G_{K}$-representation $H_{\mathrm{ett}}^{2}\left(X_{K^{\mathrm{s}}}, \mathbb{Q}_{\ell}\right)$ is unramified, and there is an isomorphism

$$
H_{\text {êt }}^{2}\left(X_{K^{s}}, \mathbb{Q}_{\ell}\right) \stackrel{\cong}{\longrightarrow} H_{\text {êt }}^{2}\left(Y_{\bar{k}}, \mathbb{Q}_{\ell}\right)
$$

of $G_{k}$-representations.

Remark. When $\operatorname{char}(k)=p>0$, there are also $p$-adic versions of both results in terms of crystalline cohomology, see [CLL17, Theorem 1.1 and Theorem 1.6].

Good reduction in positive equicharacteristic. The classical $\ell$-adic good reduction criterion of Serre-Tate for abelian varieties [ST68] makes no restriction on the generic characteristic of the base. Neither does its $p$-adic analogue [SGA7-I, Exp. IX, Thm. 5.13], phrased in terms of the pdivisible group associated to an abelian variety. The purpose of this note is therefore to explain how to extend the above results on good reduction of K3 surfaces to the case when $\operatorname{char}(K)=p>0$, both in terms of $\ell$-adic and $p$-adic cohomology. As we already observed in [CLL17], essentially everything in the $\ell$-adic case from [LM18, CLL17] goes through if Assumption $(\star)$ is strengthened 
to require the given finite extension $L / K$ to be separable - this is Assumption ( $\left.\star^{\mathrm{s}}\right)$ in $\S 2$. The key point, then, is to deal with the problem of descending good reduction over a finite and purely inseparable extension $L / K$. Our main result in this direction is the following.

Theorem (Corollary 3.6). Let $L / K$ be a finite and purely inseparable extension and $X / K$ a $K 3$ surface. Then, $X$ has good reduction over $K$ if and only if it has good reduction over $L$.

In fact, the main property of K3 surfaces needed is that they do not admit non-zero global vector fields. Thus, we actually prove a more general statement about purely inseparable descent of models (Theorem 3.5) that may be of independent interest. This result already gives the desired extensions of the above results to $\ell$-adic cohomology.

Corollary. Theorems 1 and 2 hold without any restriction on $\operatorname{char}(K)$.

In order to provide $p$-adic versions of these results in equicharacteristic $p>0$, we need to use the Robba ring valued version of rigid cohomology, as was developped in reasonable generality in [LP16]. ${ }^{1}$ Let $W=W(k)$ denote the ring of Witt vectors of $k$, write $\kappa=W[1 / p]$ for its fraction field, and $\mathcal{R}$ for the Robba ring over $\kappa$. Then for any variety $X / K$, there are rigid cohomology groups $H_{\text {rig }}^{i}(X / \mathcal{R})$, which are $(\varphi, \nabla)$-modules over $\mathcal{R}$, and behave completely analogously to the $\ell$-adic étale cohomology groups for $\ell \neq p$. In fact, since we will only be working with smooth and proper varieties it is possible to phrase everything purely in terms of crystalline cohomology, see $\S 1$ for a detailed discussion. In the $p$-adic case in equicharacteristic, the analogue of Theorem 1 is the following.

Theorem 3. Assume that $\operatorname{char}(K)=p>0$, and let $X$ be a K3 surface over $K$, satisfying Assumption $(\star)$. Consider the following statements:

(1) for some prime (resp. all primes) $\ell \neq p$, the $G_{K}$-action on $H_{\text {ét }}^{2}\left(X_{K^{\mathrm{s}}}, \mathbb{Q}_{\ell}\right)$ is unramified;

(2) the $(\varphi, \nabla)$-module $H_{\text {rig }}^{2}(X / \mathcal{R})$ admits a basis of horizontal sections;

(3) $X$ has good reduction over a finite, unramified extension $L / K$;

(4) $X$ admits a model $\mathcal{X}$ that is projective over $\mathcal{O}_{K}$, and whose special fibre is a K3 surface with at worst RDP singularities.

Then, we have $(1) \Leftrightarrow(2) \Leftrightarrow(3) \Rightarrow(4)$.

Similarly, the analogue of Theorem 2 is the following.

Theorem 4. Assume that $\operatorname{char}(K)=p>0$, and let $X$ be a K3 surface over $K$, satisfying Assumption $(\star)$. Then then following are equivalent:

(1) $X$ has good reduction over $K$;

(2) for some prime (resp. all primes) $\ell \neq p$, the $G_{K}$-representation $H_{\text {ét }}^{2}\left(X_{K^{\mathrm{s}}}, \mathbb{Q}_{\ell}\right)$ is unramified, and there is an isomorphism

$$
H_{\text {ét }}^{2}\left(X_{K^{\mathrm{s}}}, \mathbb{Q}_{\ell}\right) \stackrel{\cong}{\longrightarrow} H_{\text {ét }}^{2}\left(Y_{\bar{k}}, \mathbb{Q}_{\ell}\right)
$$

of $G_{k}$-representations.

\footnotetext{
${ }^{1}$ That this cohomology theory can be used to rephrase the 'classical' $p$-adic criterion of Grothendieck for abelian varieties is explained in $[\mathrm{LP} 16, \S 5.3]$
} 
(3) the $(\varphi, \nabla)$-module $H_{\text {rig }}^{2}(X / \mathcal{R})$ admits a basis of horizontal sections, and there is an isomorphism

$$
H_{\text {rig }}^{2}(X / \mathcal{R})^{\nabla=0} \stackrel{\cong}{\longrightarrow} H_{\text {rig }}^{2}(Y / \kappa)
$$

of $F$-isocrystals over $\kappa$.

Finally, there also exist examples similar to the ones of [LM18, Theorem 1.6] over Laurent series fields that show that in general one cannot expect to choose the finite and unramified extension $L / K$ from (3) of Theorem 3 to be trivial.

Theorem (5.1). For every prime $p \geq 5$, there exists a smooth K3 surface over $\mathbb{F}_{p}((t))$ that admits good reduction over $\mathbb{F}_{p^{2}}((t))$ but not over $\mathbb{F}_{p}((t))$.

Organisation. Let us now give a brief summary of the contents of this note. In $\S 1$ we will give a brief overview of $p$-adic cohomology over positive characteristic local fields. In $\S 2$ we will roughly outline the arguments from [LM18] and [CLL17], and explain why they carry over to the equicharacteristic $p>0$ case under a suitable strengthening of Assumption $(\star)$ that we call Assumption $\left(\star^{\mathrm{s}}\right)$. In $\S 3$ we will discuss descent under purely inseparable fpqc covers, and prove our main result that smooth models of K3 surfaces descend under purely inseparable field extensions. In $\S 4$ we will bring everything together to prove our main results, Theorems 3 and 4 . Finally, in $\S 5$ we will construct counter-examples asserted by Theorem 5.1.

Acknowledgements. We would like to thank Yuya Matsumoto for explaining to us why arithmetic threefold flops still exist in equicharacteristic 2 . The second named author is support by the Netherlands Organisation for Scientific Research (NWO). The third named author is supported by the ERC Consolidator Grant 681838 "K3CRYSTAL".

\section{REVIEW OF $p$-ADIC COHOMOLOGY IN EQUiChARACTERISTIC $p>0$}

In this section we will briefly review the facts needed on $p$-adic cohomology when $\operatorname{char}(K)=$ $p>0$. Thus, $\mathcal{O}_{K}$ is an excellent and Henselian DVR of characteristic $p>0$ with fraction field $K$ and perfect residue field $k$. We let $W=W(k)$ denote the ring of Witt vectors of $k, \kappa$ its fraction field, and $\sigma$ the $p$-power Frobenius on $W$ and $\kappa$.

Remark 1.1. The choice of $\kappa$ for the fraction field of $W(k)$ is distinctly "non-standard". However, the more usual choices of $K_{0}$ or $K$ are unfortunately excluded by the use of $K$ for our given equicharacteristic local field.

In this situation, a choice of uniformiser $\varpi \in \mathcal{O}_{K}$ induces an isomorphism $\widehat{K} \cong k((\varpi))$ between the completion of $K$ and a Laurent series field over $k$. We will denote by $\mathcal{R}$ the Robba ring over $\kappa$, that is, the ring consisting of those Laurent series $\sum_{i} a_{i} t^{i}$ with $a_{i} \in \kappa$ such that:

- for all $\rho<1,\left|a_{i}\right| \rho^{i} \rightarrow 0$ as $i \rightarrow \infty$;

- for some $\eta<1,\left|a_{i}\right| \eta^{i} \rightarrow 0$ as $i \rightarrow-\infty$.

In other words, $\mathcal{R}$ is the ring of functions convergent on some semi-open annulus $\eta \leq|t|<1$. The ring of integral elements $\mathcal{R}^{\text {int }}$ (that is, those with $a_{i} \in W$ ) is therefore a lift of $\widehat{K}$ to characteristic 0 , in the sense that mapping $t \mapsto \varpi$ induces an isomorphism $\mathcal{R}^{\text {int }} /(p) \cong \widehat{K}$. We will denote by $\sigma$ a Frobenius on $\mathcal{R}$, that is, a continuous $\sigma$-linear endomorphism preserving $\mathcal{R}^{\text {int }}$ and lifting the absolute $p$-power Frobenius on $\widehat{K}$. We will moreover assume that $\sigma(t)=u t^{p}$ for some $u \in$ 
$\left(W \llbracket t \rrbracket \otimes_{W} \kappa\right)^{\times}$. The reader is welcome to assume that $\sigma\left(\sum_{i} a_{i} t^{i}\right)=\sum_{i} \sigma\left(a_{i}\right) t^{i p}$. Let $\partial_{t}: \mathcal{R} \rightarrow \mathcal{R}$ denote the derivation given by differentiation with respect to $t$.

Definition 1.2. A $(\varphi, \nabla)$-module over $\mathcal{R}$ is a finite free $\mathcal{R}$-module $M$ together with:

- a connection, that is, a $\kappa$-linear map $\nabla: M \rightarrow M$ such that

$$
\nabla(r m)=\partial_{t}(r) m+r \nabla(m) \quad \text { for all } r \in \mathcal{R} \text { and } m \in M
$$

- a horizontal Frobenius $\varphi: \sigma^{*} M:=M \otimes_{\mathcal{R}, \sigma} \mathcal{R} \stackrel{\sim}{\rightarrow} M$.

Then, $(\varphi, \nabla)$-modules over $\mathcal{R}$ should be considered as $p$-adic analogues of Galois representations. For example, they satisfy a local monodromy theorem, see [Ked04b]. More specifically, the connection $\nabla$ should be viewed as an analogue of the action of the inertia subgroup $I_{K}$ and the Frobenius $\varphi$ the action of some Frobenius lift in $G_{K}$. The analogue for $(\varphi, \nabla)$-modules of a Galois representation being unramified is therefore the connection acting trivially, or, in other words, the $(\varphi, \nabla)$-module admitting a basis of horizontal sections.

The $p$-adic completion $\widehat{\mathcal{R}}^{\text {int }}$ of the integral Robba ring is a Cohen ring for $\widehat{K}$ and hence, any smooth and proper $\widehat{K}$-variety $Y$ has crystalline cohomology groups

$$
H_{\text {cris }}^{i}\left(Y / \widehat{\mathcal{R}}^{\text {int }}\right)
$$

which are $(\varphi, \nabla)$-modules over $\widehat{\mathcal{R}}^{\text {int }}$, see for example [GM87, §.3.1]. It follows from [Ked00, Theorem 7.0.1] (where the notations $\Gamma=\widehat{\mathcal{R}}^{\text {int }}$ and $\Gamma^{\dagger}=\mathcal{R}^{\text {int }}$ are used) that the crystalline cohomology groups of any smooth and proper variety $Y / \widehat{K}$ descend uniquely to $(\varphi, \nabla)$-modules

$$
H_{\text {cris }}^{i}\left(Y / \mathcal{R}^{\mathrm{int}}\right)
$$

over $\mathcal{R}^{\text {int }}$. Therefore, we may define for any smooth and proper variety $X / K$

$$
H_{\text {rig }}^{i}(X / \mathcal{R}):=H_{\text {cris }}^{i}\left(X_{\widehat{K}} / \mathcal{R}^{\text {int }}\right) \otimes_{\mathcal{R}^{\text {int }}} \mathcal{R}
$$

as $(\varphi, \nabla)$-modules over $\mathcal{R}$. We have the following analogue of the smooth and proper base change theorem.

Proposition 1.3. Let $\mathcal{X} \rightarrow \operatorname{Spec}\left(\mathcal{O}_{K}\right)$ be a smooth and proper morphism of algebraic spaces, whose generic fibre is a scheme. Then, for any $i \geq 0$ there exists a canonical isomorphism

$$
H_{\text {rig }}^{i}\left(\mathcal{X}_{K} / \mathcal{R}\right)^{\nabla=0} \stackrel{\cong}{\longrightarrow} H_{\text {rig }}^{i}\left(\mathcal{X}_{k} / \kappa\right),
$$

of F-isocrystals over $K$.

Proof. Since $\mathcal{X}_{k}$ is smooth over $k$, the right hand side is isomorphic to the rational log-crystalline cohomology of $\mathcal{X}_{k}$, equipped with the log structure

$$
\mathbb{N} \rightarrow \mathcal{O}_{\mathcal{X}_{k}}, \quad 1 \mapsto 0
$$

so we may apply [CL16, Proposition 2.3].

We will also need cycle class maps in $p$-adic cohomology. For any smooth and proper variety $Y / \widehat{K}$, homomorphisms

$$
[-]: \mathrm{CH}^{d}(Y) \rightarrow H_{\text {cris }}^{2 d}\left(Y / \widehat{\mathcal{R}}^{\text {int }}\right)_{\mathbb{Q}}
$$

are constructed in [GM87, Theorem 4.3.1], and since their image lands in the subspace of horizontal sections on which Frobenius acts as $p^{d}$, it follows from Kedlaya's full faithfullness theorem [Ked04a, 
Theorem 5.1] that for any smooth and proper variety $Y / \widehat{K}$, the codimension- $d$ crystalline cycle class map for $Y$ actually takes values in $H_{\text {cris }}^{2 d}\left(Y / \mathcal{R}^{\text {int }}\right)_{\mathbb{Q}}^{\nabla=0, \varphi=p^{d}}$. This allows us to define, for any smooth and proper variety $X / K$, cycle class maps

$$
[-]: \mathrm{CH}^{d}(X) \rightarrow H_{\text {rig }}^{2 d}(X / \mathcal{R})
$$

landing in the subspace of horizontal sections on which Frobenius acts as $p^{d}$. On the other hand, for any smooth and proper variety $Y / k$, we also have crystalline cycle class map

$$
[-]: \mathrm{CH}^{d}(Y) \rightarrow H_{\mathrm{rig}}^{2 d}(Y / \kappa) .
$$

The following is the $p$-adic analogue of [LM18, Lemma 5.6], see also [CCM13, $\$ 1]$.

Proposition 1.4. Let $\mathcal{X} \rightarrow \operatorname{Spec}\left(\mathcal{O}_{K}\right)$ be a smooth and proper morphism of algebraic spaces, whose special and generic fibres are schemes. Then, for any closed subspace $\mathcal{Z} \hookrightarrow \mathcal{X}$ of constant codimension $d$ and flat over $\mathcal{O}_{K}$, the isomorphism

$$
H_{\text {rig }}^{2 d}\left(\mathcal{X}_{K} / \mathcal{R}\right)^{\nabla=0} \stackrel{\cong}{\longrightarrow} H_{\text {rig }}^{2 d}\left(\mathcal{X}_{k} / \kappa\right)
$$

$\operatorname{sends}\left[\mathcal{Z}_{K}\right]$ to $\left[\mathcal{Z}_{k}\right]$

Proof. We may assume that $K=\widehat{K}$ is complete. By combining Chow's lemma with de Jong's theorem on alterations [dJ96] we may choose a finite extension $L / K$, a projective and strictly semistable scheme $\mathcal{Y} \rightarrow \operatorname{Spec}\left(\mathcal{O}_{L}\right)$ and an alteration $\pi: \mathcal{Y} \rightarrow \mathcal{X}$ of algebraic spaces over $\mathcal{O}_{K}$.

Let $k_{L}$ denote the residue field of $L, W_{L}=W\left(k_{L}\right)$ and fix a lift of $\mathcal{O}_{K} \rightarrow \mathcal{O}_{L}$ of the form $W \llbracket t \rrbracket \rightarrow W_{L} \llbracket t_{L} \rrbracket$. Let $\mathcal{R}_{L}$ denote a copy of the Robba ring over $\kappa_{L}=W_{L}[1 / p]$ with parameter $t_{L}$, and $\mathcal{R} \rightarrow \mathcal{R}_{L}$ the induced finite flat extension. This sends $\mathcal{R}^{\text {int }}$ into $\mathcal{R}_{L}^{\text {int }}$. Let $\mathcal{Y}^{\times}$denote the scheme $\mathcal{Y}$ equipped with the fs $\log$ structure induced by the special fibre, and $\mathcal{Y}_{k_{L}}^{\times}$the special fibre of this $\log$ scheme. Let $W_{k_{L}}^{\times}$denote the ring $W_{k_{L}}$ equipped with the log structure

$$
\begin{aligned}
\mathbb{N} & \rightarrow W_{k_{L}} \\
1 & \mapsto 0
\end{aligned}
$$

and $W_{L} \llbracket t_{L} \rrbracket^{\times}$for $W_{L} \llbracket t_{L} \rrbracket$ equipped with the log structure

$$
\begin{aligned}
\mathbb{N} & \rightarrow W_{k_{L}} \llbracket t_{L} \rrbracket \\
1 & \mapsto t_{L} .
\end{aligned}
$$

By [LP16, Theorem 5.46] there is, for all $i \geq 0$, an isomorphism

$$
H_{\text {rig }}^{i}\left(\mathcal{Y}_{L} / \mathcal{R}_{L}\right)^{\nabla=0} \stackrel{\cong}{\longrightarrow} H_{\log \text {-cris }}^{i}\left(\mathcal{Y}_{k_{L}}^{\times} / W_{L}^{\times}\right) \otimes \mathbb{Q}
$$

fitting into a commutative diagram

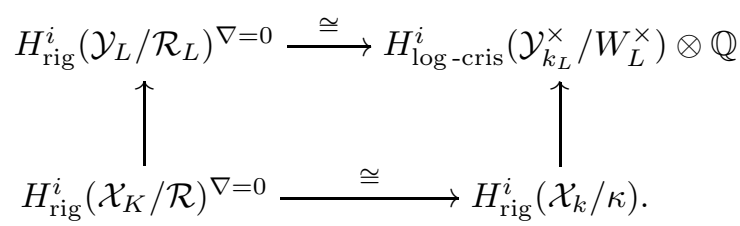

Both vertical maps are injective by Poincaré duality (in the log crystalline case, see [Tsu99]). In particular, it suffices to verify that $\left[\mathcal{Z}_{K}\right]$ and $\left[\mathcal{Z}_{k}\right]$ map to the same element in $H_{\log \text {-cris }}^{2 d}\left(\mathcal{Y}_{k_{L}}^{\times} / W_{L}\right) \otimes \mathbb{Q}$. 
For any algebraic space over $\mathcal{O}_{K}$, we will let $K(-)$ denote the $K$-theory of vector bundles. We therefore have total Chern class maps

$$
\begin{aligned}
& c: K_{0}\left(\mathcal{X}_{K}\right) \rightarrow \bigoplus_{n \geq 0} H_{\mathrm{cris}}^{2 n}\left(\mathcal{X}_{K} / \widehat{\mathcal{R}}^{\mathrm{int}}\right) \\
& c: K_{0}\left(\mathcal{Y}_{K}\right) \rightarrow \bigoplus_{n \geq 0} H_{\mathrm{cris}}^{2 n}\left(\mathcal{Y}_{K} / \widehat{\mathcal{R}}_{L}^{\mathrm{int}}\right) \\
& c: K_{0}\left(\mathcal{X}_{k}\right) \rightarrow \bigoplus_{n \geq 0} H_{\mathrm{cris}}^{2 n}\left(\mathcal{X}_{k} / W\right)
\end{aligned}
$$

taking values in crystalline cohomology, as constructed in [BI70]. Using exactly the same method as in [BI70] we can also construct total Chern class maps

$$
\begin{aligned}
c: K_{0}(\mathcal{Y}) & \rightarrow \bigoplus_{n \geq 0} H_{\log \text {-cris }}^{2 n}\left(\mathcal{Y}^{\times} / W_{L} \llbracket t_{L} \rrbracket^{\times}\right) \\
c: K_{0}\left(\mathcal{Y}_{k_{L}}\right) & \rightarrow \bigoplus_{n \geq 0} H_{\log \text {-cris }}^{2 n}\left(\mathcal{Y}_{k_{L}}^{\times} / W_{L}^{\times}\right)
\end{aligned}
$$

in log-crystalline cohomology. Indeed, if we have a $p$-adic log PD base $S$, (e.g. $W^{\times}$or $W \llbracket t \rrbracket^{\times}$) and an fs $\log$ scheme $X$ equipped with a $\log$ smooth and proper morphism $X \rightarrow S$, then for any rank $r$ vector bundle $\mathcal{E}$ on $X$, we can endow its projectivisation $g: \mathbb{P}(\mathcal{E}) \rightarrow X$ with the pullback log-structure from $X$. The first Chern class

$$
\xi:=c_{1}\left(\mathcal{O}_{\mathbb{P}(\mathcal{E})}(1)\right) \in H_{\log -\operatorname{cris}}^{2}(\mathbb{P}(\mathcal{E}) / W)
$$

can be defined directly, and in order to follow the contruction in [BI70] we need to know that the cohomology $H_{\log \text {-cris }}^{*}(\mathbb{P}(\mathcal{E}) / W)$ decomposes as a direct sum

$$
H_{\log \text {-cris }}^{*}(\mathbb{P}(\mathcal{E}) / S) \cong \bigoplus_{i=0}^{r-1} H_{\log \text {-cris }}^{*}(X / S) \cdot \xi^{i} .
$$

We can turn this into a local statement, namely that the higher direct image $\mathbf{R} g_{*} \mathcal{O}_{\mathbb{P}(\mathcal{E}) / S}^{\log \text {-cris }}$ decomposes as

$$
\mathbf{R} g_{*} \mathcal{O}_{\mathbb{P}(\mathcal{E}) / S}^{\log \text {-cris }} \cong \bigoplus_{i=0}^{r-1} \mathcal{O}_{X / S}^{\log \text {-cris }} \cdot \xi^{i}
$$

where we have abused notation and also written $\xi \in H_{\log \text {-cris }}^{0}\left(X / S, \mathbf{R}^{2} g_{*} \mathcal{O}_{\mathbb{P}(\mathcal{E}) / S}^{\log \text {-cris }}\right)$ for the image of the first Chern class of $\mathcal{O}_{\mathbb{P}(\mathcal{E})}(1)$ under the edge morphism of the Leray spectral sequence. Since this statement is now local, we can assume that in fact $\mathcal{E}=\mathcal{O}_{X}^{\oplus r}$, thus $\mathbb{P}(\mathcal{E})=\mathbb{P}_{X}^{r-1}$. In this case the claim follows from the smooth and proper base change theorem in log-crystalline cohomology [Kat89, Theorem 6.10], together with the standard computation of the crystalline cohomology of projective space.

Now, since $\mathcal{Z}$ is flat, the fibres $\mathcal{Z}_{K}$ and $\mathcal{Z}_{k}$ are also of codimension $d$ in $\mathcal{X}_{K}$ and $\mathcal{X}_{k}$ respectively. Since $\mathcal{X}_{K}$ and $\mathcal{X}_{k}$ are both regular and projective schemes, the $K$-theory of vector bundles coincides with that of coherent sheaves, so we have well-defined classes $\left[\mathcal{O}_{\mathcal{Z}_{K}}\right] \in K_{0}\left(\mathcal{X}_{K}\right)$ and $\left[\mathcal{O}_{\mathcal{Z}_{k}}\right] \in K_{0}\left(\mathcal{X}_{k}\right)$. Essentially by definition [GM87, Theorem 4.3.1], we have

$$
\left[\mathcal{Z}_{K}\right]=\frac{(-1)^{d-1}}{(d-1) !} c_{d}\left(\left[\mathcal{O}_{\mathcal{Z}_{K}}\right]\right) \quad \text { and } \quad\left[\mathcal{Z}_{k}\right]=\frac{(-1)^{d-1}}{(d-1) !} c_{d}\left(\left[\mathcal{O}_{\mathcal{Z}_{k}}\right]\right)
$$


Since $\mathcal{O}_{\mathcal{Z}_{K}}$ and $\mathcal{O}_{Z_{k}}$ are strictly perfect complexes on $\mathcal{X}_{K}$ and $\mathcal{X}_{k}$ respectively, we get well-defined classes $\left[\mathbf{L} \pi^{*} \mathcal{O}_{\mathcal{Z}_{K}}\right] \in K_{0}\left(\mathcal{Y}_{L}\right)$ and $\left[\mathbf{L} \pi^{*} \mathcal{O}_{\mathcal{Z}_{k}}\right] \in K_{0}\left(\mathcal{Y}_{k_{L}}\right)$. By functoriality of Chern classes, it suffices to show that the isomorphism

$$
H_{\text {rig }}^{2 d}\left(\mathcal{Y}_{L} / \mathcal{R}_{L}\right)^{\nabla=0} \stackrel{\cong}{\longrightarrow} H_{\log \text {-cris }}^{2 d}\left(\mathcal{Y}_{k_{L}}^{\times} / W_{L}^{\times}\right) \otimes \mathbb{Q}
$$

sends $c_{d}\left(\left[\mathbf{L} \pi^{*} \mathcal{O}_{\mathcal{Z}_{K}}\right]\right)$ to $c_{d}\left(\left[\mathbf{L} \pi^{*} \mathcal{O}_{\mathcal{Z}_{k}}\right]\right)$. Since $\mathcal{X}$ is regular we know that $\mathcal{O}_{\mathcal{Z}}$ is a perfect complex of $\mathcal{O}_{\mathcal{X}}$-modules, and since $\mathcal{Y}$ is a projective scheme, $\mathbf{L} \pi^{*} \mathcal{O}_{\mathcal{Z}}$ is actually a strictly perfect complex on $\mathcal{Y}$. It therefore has a well defined class in $K_{0}(\mathcal{Y})$, which restricts to the class of $\mathbf{L} \pi^{*} \mathcal{O}_{\mathcal{Z}_{K}}$ (resp. $\mathbf{L} \pi^{*} \mathcal{O}_{\mathcal{Z}_{k}}$ ) on the generic (resp. special) fibre. Given the construction of the isomorphism

$$
H_{\text {rig }}^{2 d}\left(\mathcal{Y}_{L} / \mathcal{R}_{L}\right)^{\nabla=0} \stackrel{\cong}{\longrightarrow} H_{\text {log -cris }}^{2 d}\left(\mathcal{Y}_{k_{L}}^{\times} / W_{L}^{\times}\right) \otimes \mathbb{Q}
$$

it now simply suffices to note that the diagram

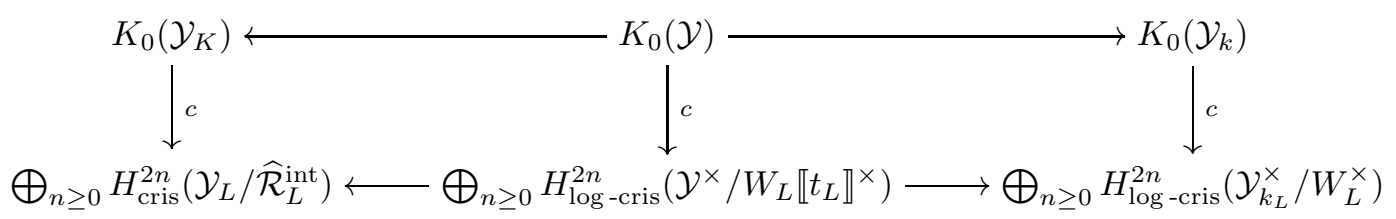

commutes.

Remark 1.5. We need to argue on the alteration $\mathcal{Y}$ rather than on the model $\mathcal{X}$ itself because of the potential difference between the $K$-group of vector bundles (equivalently: strictly perfect complexes) and that of perfect complexes. They coincide on regular schemes (like $\mathcal{Y}$ ), but it is not known whether the same is true for algebraic spaces (like $\mathcal{X}$ ). If it were, the above proof could be significantly simplified.

\section{Descending Good REDUCTION Under SEPARABle EXtensions}

In this section, we will explain how and why the main results of [LM18] and [CLL17] carry over in equicharacteristic $p$ under a strengthening of Assumption $(\star)$, which we will call Assumption $\left(\star^{\mathrm{s}}\right)$. Under this stronger assumption, we also establish a version of these results in terms of the Robba ring valued $p$-adic cohomology discussed in $\S 1$.

Assumption $\left(\star^{\mathrm{s}}\right)$. A K3 surface $X / K$ satisfies $\left(\star^{\mathrm{s}}\right)$ if it satisfies Assumption $(\star)$ and we can moreover take the finite extension $L$ to be separable over $K$.

Then we can use the arguments of [LM18] word-for-word to prove the following.

Theorem 2.1. Let $X$ be a $K 3$ surface over $K$, satisfying Assumption $\left(\star^{\mathrm{s}}\right)$, and let $\ell$ be a prime $\neq p$. Consider the following three statements:

(1) the $G_{K}$-action on $H_{\mathrm{et}}^{2}\left(X_{K^{\mathrm{s}}}, \mathbb{Q}_{\ell}\right)$ is unramified;

(2) $X$ has good reduction over a finite, unramified extension $L / K$;

(3) $X$ admits a model $\mathcal{X}$ that is projective over $\mathcal{O}_{K}$, and whose special fibre is a K3 surface with at worts RDP singularities.

Then, we have $(1) \Leftrightarrow(2) \Rightarrow(3)$. 
Proof. The point is that the characteristic 0 hypothesis is only used in [LM18] in order to ensure that $X$ admits a Kulikov model over a Galois extension $L / K$, which enables arguments via Galois descent to be used. Thus, if we admit this from the start, the proofs in [LM18] go through unchanged, with one important exception.

This exception occurs in the proof of [LM18, Proposition 4.2], showing the existence of flops on relative surfaces over $\mathcal{O}_{K}$ with numerically trivial relative canonical divisor. One step of the construction uses the fact that a deformation

$$
\operatorname{Spf}\left(\frac{\mathcal{O}_{K} \llbracket x, y, z \rrbracket}{z^{2}-H_{1}(x, y) z-H_{0}(x, y)}\right)
$$

of a rational double point singularity over $k$ admits a non-trivial involution

$$
z \mapsto H_{1}(x, y)-z .
$$

This will clearly remain true provided $\operatorname{char}(K) \neq 2$, and to show that it also remains true when $\operatorname{char}(K)=2$, we need to explain why we will always have $H_{1}(x, y) \neq 0$.

This was pointed out to us by Yuya Matsumoto. Indeed, suppose that we have $H_{1}(x, y)=0$. Then, since the equation

$$
\frac{\partial H_{0}}{\partial x}=\frac{\partial H_{0}}{\partial y}=0
$$

has the solution $(x, y)=(0,0)$ on the special fibre, after possibly replacing $K$ by a finite extension, it also has a solution $(x, y)=(\xi, \eta)$ on the generic fibre. This would mean that the generic fibre has a singular point $(x, y, z)=\left(\xi, \eta, \sqrt{H_{0}(\xi, \eta)}\right)$. Therefore smoothness of the generic fibre means that we must have $H_{1}(x, y) \neq 0$ after all.

In order to have a version of this result also in terms of the Robba ring valued $p$-adic cohomology discussed in $\S 1$, the first step is to have an analogue of [Mat15, Theorem 1.1] in this cohomology theory. This is provided by [CL16, Theorem 6.4]. The next key point will be to show that smooth models descend under finite, totally ramified extensions, under suitable cohomological assumptions. More precisely, we need to establish the analogue of [LM18, Proposition 5.8] in Robba-ring value rigid cohomology.

Thus, let $X$ be a K3 surface over $K$. Suppose that there exists a totally ramified Galois extension $L / K$ with Galois group $G$ such that $X_{L}$ admits a smooth model $\mathcal{X} \rightarrow \operatorname{Spec}\left(\mathcal{O}_{L}\right)$. Let $\mathcal{R}$ be the Robba ring over $\kappa$ and let $\mathcal{R}_{L}$ denote the unique unramified extension of $\mathcal{R}$ corresponding to $L / K$ as in [Mat95], see also the proof of Proposition 1.4. Then, there is a natural $G$-action on the $(\varphi, \nabla)$-module $H_{\text {rig }}^{2}\left(X_{L} / \mathcal{R}_{L}\right)$ and hence, on its subspace $H_{\text {rig }}^{2}\left(X_{L} / \mathcal{R}_{L}\right)^{\nabla=0}$ of horizontal sections.

Proposition 2.2. Under the previous assumptions, assume that the $G$-action on $H_{\text {rig }}^{2}\left(X_{L} / \mathcal{R}_{L}\right)^{\nabla=0}$ is trivial. Then, the $G$-action on $X_{L}$ extends to $\mathcal{X}$ and the induced $G$-action on the special fibre $\mathcal{X}_{k}$ is trivial.

Proof. The key ingredient here is Proposition 1.4. Indeed, given this we can copy the proof of [LM18, Proposition 5.5] word for word to show that the $G$-action on $X_{L}$ extends to $\mathcal{X}$. To show that the induced action on the special fibre is trivial, we use [Ogu79, Corollary 2.5] and [Keu16, Theorem 1.4], which give injectivity of the natural map

$$
\operatorname{Aut}(Y) \rightarrow \mathrm{GL}\left(H_{\text {rig }}^{2}(Y / \kappa)\right)
$$

for K3 surfaces over $k$. 
After this preparation, we arrive at the following $p$-adic version of Theorem 2.1.

Theorem 2.3. Assume that $\operatorname{char}(K)=p>0$, let $X$ be a $K 3$ surface over $K$, satisfying Assumption $\left(\star^{\mathrm{S}}\right)$, and let $\ell$ be a prime $\neq p$. Then (1) and (2) in Theorem 2.1 are both equivalent to the following:

$(1)^{\prime}$ the $(\varphi, \nabla)$-module $H_{\text {rig }}^{2}(X / \mathcal{R})$ admits a basis of horizontal sections.

Proof. The implication $(2) \Rightarrow(1)^{\prime}$ follows from Proposition 1.3, together with the fact that a $(\varphi, \nabla)$ module $M$ over $\mathcal{R}$ admits a basis of horizontal sections if and only if it does so after replacing $\kappa$ by a finite extension.

For the implication $(1)^{\prime} \Rightarrow(2)$, the main extra input we need is the correct analogue of [LM18, Proposition 5.8], which is provided by Proposition 2.2. The completion of the proof of Theorem 2.3 is now geometric and identical to the $\ell$-adic case.

We can also extend the results of [CLL17] to equicharacteristic with essentially no difficulties.

Theorem 2.4. Assume that $\operatorname{char}(K)=p>0$ and let $X$ be a K3 surface over $K$, satisfying Assumption $\left(\star^{\mathrm{s}}\right)$. Then, then following are equivalent:

(1) $X$ has good reduction over $K$;

(2) for some prime (resp. all primes) $\ell \neq p$, the $G_{K}$-representation $H_{\text {èt }}^{2}\left(X_{K^{\mathrm{s}}}, \mathbb{Q}_{\ell}\right)$ is unramified, and there is an isomorphism

$$
H_{\text {èt }}^{2}\left(X_{K^{\mathrm{s}}}, \mathbb{Q}_{\ell}\right) \stackrel{\cong}{\longrightarrow} H_{\text {èt }}^{2}\left(Y_{\bar{k}}, \mathbb{Q}_{\ell}\right)
$$

of $G_{k}$-representations.

(3) the $(\varphi, \nabla)$-module $H_{\text {rig }}^{2}(X / \mathcal{R})$ admits a basis of horizontal sections, and there is an isomorphism

$$
H_{\text {rig }}^{2}(X / \mathcal{R})^{\nabla=0} \stackrel{\cong}{\longrightarrow} H_{\text {rig }}^{2}(Y / \kappa)
$$

of $F$-isocrystals over $\kappa$.

Proof. The existence of flops that is required in [CLL17, Proposition 7.5], follows from the same argument we used during the proof of Theorem 2.1. Apart from this, everything in [CLL17, §7] works exactly the same in positive equicharacteristic. The same is true for [CLL17, §8], replacing $\mathbb{D}_{\text {cris }}\left(H_{\text {ét }}^{2}\left(X_{\bar{K}}, \mathbb{Q}_{p}\right)\right)$ everywhere by $H_{\text {rig }}^{2}(X / \mathcal{R})^{\nabla=0}$, and using Proposition 1.4 in order to prove the correct analogue of [CLL17, Lemma 8.1]. Everything in [CLL17, §9] goes through unchanged.

\section{DESCENDING GOOD REDUCTION UNDER PURELY INSEPARABLE EXTENSIONS}

In this section, we shall show that smooth models of K3 surfaces descend under purely inseparable extensions of $K$, although we will start off in rather more generality than that. Let $S$ be a scheme and let $p: S^{\prime} \rightarrow S$ be a purely inseparable (that is, radicial, or universally injective) fpqc covering.

Lemma 3.1. The map $p: S^{\prime} \rightarrow S$ is separated and the diagonal embedding $\Delta: S^{\prime} \rightarrow S^{\prime} \times{ }_{S} S^{\prime}$ is a nilpotent closed immersion.

Proof. Since $p$ is surjective and universally injective, it is universally bijective. In particular, we see that either projection $p_{i}: S^{\prime} \times_{S} S^{\prime} \rightarrow S^{\prime}$ is bijective. Since $\Delta$ is a section of $p_{i}$, we deduce that $\Delta$ and $p_{i}$ are in fact both homeomorphisms. As $\Delta$ is a locally closed embedding with closed image, it 
must therefore be a closed immersion, and $p$ is therefore separated. Since $\Delta$ is a closed immersion inducing a homeomorphism on the underlying topological spaces, it is defined by a nilpotent sheaf of ideals.

We denote by $\mathcal{I}$ the ideal of the diagonal $S^{\prime} \hookrightarrow S^{\prime} \times{ }_{S} S^{\prime}$, and write

$$
S^{\prime(n)}:=\underline{\operatorname{Spec}}_{S^{\prime} \times S_{S}}\left(\mathcal{O}_{S^{\prime} \times S_{S} S^{\prime}} / \mathcal{I}^{n+1}\right)
$$

for the $n$th infinitesimal neighbourhood of $S^{\prime}$ in $S^{\prime} \times{ }_{S} S^{\prime}$. Let $f: Y \rightarrow S^{\prime}$ be an algebraic space with relative tangent sheaf $\mathcal{T}_{Y / S^{\prime}}:=\mathcal{H}_{\mathrm{om}_{\mathcal{O}_{Y}}}\left(\Omega_{Y / S^{\prime}}^{1}, \mathcal{O}_{Y}\right) \in \mathrm{QCoh}(Y)$. For any morphism of schemes $U \rightarrow S$ we write $(-)_{U}$ for the fibre product $(-) \times{ }_{S} U$.

Proposition 3.2. Assume that:

(1) the ideal $\mathcal{I}$ is of finite type;

(2) each $S^{\prime(n)}$ is flat over $S^{\prime}$ (under either projection);

(3) $Y$ is smooth and separated over $S^{\prime}$;

(4) $f_{*} \mathcal{T}_{Y / S^{\prime}}=0$, and $\mathbf{R}^{1} f_{*} \mathcal{T}_{Y / S^{\prime}}$ is torsion free.

Then, for any dense open subscheme $U \subset S$, the function

$$
\left\{\text { descent data on } Y \text { along } S^{\prime} \rightarrow S\right\} \rightarrow\left\{\text { descent data on } Y_{U} \text { along } S_{U}^{\prime} \rightarrow U\right\}
$$

obtained by restriction is a bijection.

Remark 3.3. Note that we do not assume $Y$ proper over $S^{\prime}$. However, the condition that $f_{*} \mathcal{T}_{Y / S^{\prime}}=$ 0 will generally force this in practice.

Proof. Write $p_{i}^{*}$ for pullback along either projection $p_{i}: S^{\prime} \times{ }_{S} S^{\prime} \rightarrow S^{\prime}$. The given function is injective since $Y$ is separated over $S^{\prime}$, thus an isomorphism $p_{1}^{*} Y_{U} \rightarrow p_{2}^{*} Y_{U}$ can extend to at most one isomorphism $p_{1}^{*} Y \stackrel{\sim}{\rightarrow} p_{2}^{*} Y$. To see that it is surjective, it suffices to show that we can always extend such an isomorphism (the fact that such an extension will define a descent datum again follows from separatedness of $Y \rightarrow S^{\prime}$ and the corresponding fact for $Y_{U}$ ). Since $S^{\prime} \rightarrow S^{\prime} \times{ }_{S} S^{\prime}$ is a homeomorphism, $S^{\prime} \times{ }_{S} S^{\prime}$ is covered by open sets of the form $V \times{ }_{S} V$ with $V \subset S^{\prime}$ open and affine. Therefore appealing once more to the fact that $U \subset S$ is dense and $Y \rightarrow S^{\prime}$ is separated, we can see that the problem of extending $\alpha_{U}$ is local on both $S$ and $S^{\prime}$, which we may therefore assume to be affine, say $S=\operatorname{Spec}(R)$ and $S^{\prime}=\operatorname{Spec}\left(R^{\prime}\right)$. (We may have lost surjectivity of $S^{\prime} \rightarrow S$ but this doesn't matter). We may also assume that $U=\operatorname{Spec}\left(R\left[g^{-1}\right]\right)$ is a basic open affine in $S$.

We will prove that any such $\alpha_{U}: p_{1}^{*} Y_{U} \rightarrow p_{2}^{*} Y_{U}$ extends by using deformation theory. Let $R^{\prime \prime}:=R^{\prime} \otimes_{R} R^{\prime}$ and let $I=\operatorname{ker}\left(R^{\prime \prime} \rightarrow R^{\prime}\right)$. Thus, $I$ is a nilpotent ideal. By assumption, it is finitely generated and hence, $I^{N}=0$ for $N$ large enough. If we write $R^{\prime(n)}:=R^{\prime \prime} / I^{n+1}$, then by assumption each $R^{\prime(n)}$ is flat over $R$, hence so is the kernel $I^{n} / I^{n+1}$ of the surjection $R^{\prime(n)} \rightarrow R^{\prime(n-1)}$. Let $p_{i}^{(n) *} Y$ denote the base change to $R^{\prime(n)}$ along either 'projection' map $p_{i}^{(n)}: R^{\prime} \rightarrow R^{\prime(n)}$. We will show by induction on $n \geq 1$ that $\alpha_{U}^{(n)}: p_{1}^{(n) *} Y_{U} \stackrel{\sim}{\rightarrow} p_{2}^{(n) *} Y_{U}$ extends to an isomorphism $p_{1}^{(n) *} Y \stackrel{\sim}{\rightarrow} p_{2}^{(n) *} Y$. Since $I^{N}=0$ for large enough $N$, this suffices to prove the result.

The case $n=0$ is trivial and we may therefore suppose that $n \geq 1$ and that we are given $p_{1}^{(n-1) *} Y \stackrel{\sim}{\rightarrow} p_{2}^{(n-1) *} Y$ extending $\alpha_{U}^{(n-1)}$. We can thus view both $p_{1}^{(n) *} Y$ and $p_{2}^{(n) *} Y$ as deformations of $p_{1}^{(n-1) *} Y$ along $R^{\prime(n)} \rightarrow R^{\prime(n-1)}$. By flatness of $I^{n} / I^{n+1}$ over $R^{\prime}$, the isomorphism classes of 
such deformations are controlled by

$$
H^{1}\left(Y, \mathcal{T}_{Y / R^{\prime}}\right) \otimes_{R^{\prime}} I^{n} / I^{n+1},
$$

and we are given that these two classes map to the same element in

$$
H^{1}\left(Y, \mathcal{T}_{Y / R^{\prime}}\right) \otimes_{R^{\prime}} I^{n} / I^{n+1} \otimes_{R} R\left[g^{-1}\right] .
$$

But by the torsion-free hypothesis on $\mathbf{R}^{1} f_{*} \mathcal{T}_{Y / S^{\prime}}$, we know that

$$
H^{1}\left(Y, \mathcal{T}_{Y / R^{\prime}}\right) \otimes_{R^{\prime}} I^{n} / I^{n+1} \hookrightarrow H^{1}\left(Y, \mathcal{T}_{Y / R^{\prime}}\right) \otimes_{R^{\prime}} I^{n} / I^{n+1} \otimes_{R} R\left[g^{-1}\right]
$$

is injective, from which we deduce that $p_{1}^{(n) *} Y$ and $p_{2}^{(n) *} Y$ are isomorphic as deformations of $p_{1}^{(n-1) *} Y$. Moreover, the automorphism group of a given deformation of $p_{1}^{*(n-1)} Y_{U}$ is

$$
H^{0}\left(Y, \mathcal{T}_{Y / R^{\prime}}\right) \otimes_{R^{\prime}} I^{n} / I^{n+1} \otimes_{R} R\left[g^{-1}\right]=0,
$$

since $f_{*} \mathcal{T}_{Y / S^{\prime}}=0$, from which we deduce that any isomorphism $p_{1}^{(n) *} Y \stackrel{\sim}{\rightarrow} p_{2}^{*(n)} Y$ as deformations of $p_{1}^{(n-1) *} Y$ restricts to the given isomorphism $\alpha_{U}^{(n)}: p_{1}^{(n) *} Y_{U} \stackrel{\sim}{\rightarrow} p_{2}^{(n) *} Y_{U}$.

Concerning effectivity of descent, we have the following, presumably well-known, result.

Proposition 3.4. For any algebraic space $Y \rightarrow S^{\prime}$, all descent data on $Y$ along $p: S^{\prime} \rightarrow S$ are effective.

Proof. This is entirely straightforward for schemes and not much harder for algebraic spaces. Let $Y \cong[R \rightrightarrows U]$ be a presentation of $Y$ via an étale equivalence relation of affine schemes over $S^{\prime}$. Let $\alpha: p_{1}^{*} Y \stackrel{\sim}{\rightarrow} p_{2}^{*} Y$ be an isomorphism defining a descent datum on $Y$. Since the diagonal $S^{\prime} \rightarrow S^{\prime} \times{ }_{S} S^{\prime}$ is a nilpotent closed immersion, it is a universal homeomorphism, and we can deduce from topological invariance of the small étale site (in particular, from the equivalence $\operatorname{Ét}(Y) \cong \operatorname{Ét}\left(p_{i}^{*} Y\right)$ for each $\left.i\right)$ that we can uniquely extend $\alpha$ to an isomorphism

$$
p_{1}^{*} R \rightrightarrows p_{1}^{*} U \cong p_{2}^{*} R \rightrightarrows p_{2}^{*} U
$$

of étale equivalence relations. This isomorphism is compatible on $S^{\prime} \times{ }_{S} S^{\prime} \times_{S} S^{\prime}$ and hence (since $U$ and $R$ are affine), descends to an étale equivalence relation $R_{S} \rightrightarrows U_{S}$ of affine schemes over $S$. Thus, taking $X \cong\left[R_{S} \rightrightarrows U_{S}\right]$ to be the corresponding quotient we get the required descent of $Y$ to $S$.

We will apply these results as follows. Let $\mathcal{O}_{K}$ be our fixed excellent and Henselian DVR with fraction field $K$. Let $X$ be a smooth, proper, and geometrically connected scheme over $K$ and $L / K$ a finite and purely inseparable extension. The integral closure $\mathcal{O}_{L}$ of $\mathcal{O}_{K}$ inside $L$ is then also an excellent and Henselian DVR. Suppose that we have a smooth model $\mathcal{Y}$ for $X_{L}$ over $\mathcal{O}_{L}$.

Theorem 3.5. Assume that $H^{0}\left(X, \mathcal{T}_{X / K}\right)=0$ and that $H^{1}\left(\mathcal{Y}, \mathcal{T}_{\mathcal{Y} / \mathcal{O}_{L}}\right)$ is torsion free. Then, $\mathcal{Y}$ descends uniquely to a smooth model for $X$ over $\mathcal{O}_{K}$.

Proof. Since $X_{L}$ comes with a canonical descent datum, we apply Proposition 3.2 with $S=$ $\operatorname{Spec}\left(\mathcal{O}_{K}\right), S^{\prime}=\operatorname{Spec}\left(\mathcal{O}_{L}\right)$ and $U=\operatorname{Spec}(K)$ to extend this descent datum to $\mathcal{Y}$, and then apply Propostion 3.4 to deduce that this descent datum is effective.

Since K3 surfaces do not admit non-zero global vector fields [RŠ76], the previous result applies to them: 
Corollary 3.6. Suppose that $X$ is a K3 surface over $K$. Then, any smooth model for $X_{L}$ descends uniquely to a smooth model for $X$. In particular, $X$ has good reduction over $K$ if and only if $X_{L}$ has good reduction over $L$.

\section{MAin RESUlts}

Using the results of $\S 3$, it is now straightforward to deduce our main results, via the following proposition.

Proposition 4.1. Suppose that $\operatorname{char}(K)=p>0$ and let $X$ be a K3 surface over $K$. Assume that either of the following conditions holds:

(1) for some prime $\ell \neq p$ the $G_{K}$-representation $H_{\text {ét }}^{2}\left(X_{K^{\mathrm{s}}}, \mathbb{Q}_{\ell}\right)$ is unramified;

(2) the $(\varphi, \nabla)$-module $H_{\text {rig }}^{2}(X / \mathcal{R})$ admits a basis of horizontal sections.

Then, $X$ satisfies Assumption ( $\star$ ) if and only if it satisfies Assumption $\left(\star^{\mathrm{s}}\right)$.

Proof. We clearly have that Assumption $\left(\star^{\mathrm{s}}\right)$ implies Assumption $(\star)$.

For the converse direction, let $L / K$ be a finite extension over which $X$ admits a Kulikov model. Note that either condition of the proposition also holds for the base change $X_{L}$ and hence, by [CL16, Theorem 6.4] we know that $X_{L}$ has good reduction. If we let $K \subset K_{1} \subset L$ denote the maximal separable subextension, it therefore follows from Corollary 3.6 that $X_{K_{1}}$ has good reduction. In particular, $X$ admits a Kulikov model over $K_{1}$ and thus, satisfies Assumption ( $\star$ ).

Applying the results of $\S 2$, Theorems 3 and 4 now follow immediately.

\section{A COUnTER-EXAMPle}

In this section, we will explain how to modify the example given in [LM18, §7] to produce K3 surfaces in equicharacteristic $p>0$ that only admit good reduction after a non-trivial finite and unramified extension. Let $p \geq 5$, choose $c \in \mathbb{F}_{p}^{*} \backslash\left(\mathbb{F}_{p}^{*}\right)^{2}, a \in \mathbb{F}_{p}^{*} \backslash\left\{\frac{16}{27}\right\}$ and let $F \in \mathbb{F}_{p}[t][x, y, z, w]$ be the polynomial

$$
F:=w\left(x^{3}+y^{3}+z^{3}+(t+a(1-t)) w^{3}\right)+\left(t z^{2}+x y+t y z\right)^{2}-(c-t) t^{2} y^{2} z^{2} .
$$

Define $\mathcal{X}:=V(F) \subset \mathbb{P}_{\mathbb{F}_{p} \llbracket t \rrbracket}^{3}$ and let $X=\mathcal{X}_{\mathbb{F}_{p}((t))}$ be its generic fibre.

Theorem 5.1. $X$ is a smooth K3 surface over $\mathbb{F}_{p}((t))$ that admits good reduction over $\mathbb{F}_{p^{2}}((t))$ but not over $\mathbb{F}_{p}((t))$.

Proof. Exactly as in [LM18, Theorem 7.2], we can verify smoothness of $X$ by considering the subscheme cut out by $F$ in $\mathbb{P}_{\mathbb{F}_{p}[t]}^{3}$ and reducing modulo $1-t$. The special fibre of $\mathcal{X}$ is isomorphic to the special fibre of the example $\mathcal{X}(p)$ constructed in [LM18], it is therefore a singular K3 surface with 6 RDP singularities, two of which are defined over $\mathbb{F}_{p}$ and the other four over $\mathbb{F}_{p}\left[\zeta_{3}\right]$. Since $\sqrt{c-t} \in \mathbb{F}_{p^{2}} \llbracket t \rrbracket$, after extending scalars to $\mathbb{F}_{p^{2}} \llbracket t \rrbracket$ we can resolve the singularities of $\mathcal{X}$ by blowing up either ideal

$$
\mathcal{I}_{ \pm}=\left(w, t z^{2}+x y+t y z \pm t y z \sqrt{c-t}\right) .
$$

However, these two different ideals give rise to distinct elements of the strictly local Picard group $\operatorname{Pic}\left(\mathcal{O}_{\mathcal{X}, \bar{x}}^{\text {sh }}\right)$, which are interchanged by the action of $\left.\operatorname{Gal}\left(\mathbb{F}_{p}((t))\right)^{\mathrm{s}} / \mathbb{F}_{p}((t))\right)$. Thus, exactly as in $[\operatorname{LM} 18$, Theorem 7.2], $X$ cannot have a smooth model over $\mathbb{F}_{p} \llbracket t \rrbracket$. 


\section{REFERENCES}

[BI70] P. Berthelot and L. Illusie, Classes de Chern en cohomologie cristalline, C. R. Acad. Sci. Paris Sér. A-B 270270 (1970), A1750-A1752. $\uparrow 7$

[CCM13] B. Chiarellotto, A. Ciccioni, and N. Mazzari, Cycle classes and the syntomic regulator, Algebra Number Theory 7 (2013), no. 3, 533-566, http://dx.doi.org/10.2140/ant.2013.7.533. $\uparrow 6$

[CL16] B. Chiarellotto and C. Lazda, Combinatorial degenerations of surfaces and Calabi-Yau threefolds, Algebra Number Theory 10 (2016), no. 10, 2235-2266, http://dx.doi.org/10.2140/ant.2016.10.2235. 个 5, 9, 13

[CLL17] B. Chiarellotto, C. Lazda, and C. Liedtke, A Néron-Ogg-Shafarevich criterion for K3 surfaces, preprint (2017), https://arXiv.org/abs/1701.02945/, to appear in Proc. London Math. Soc. $\uparrow 1,2,4,8,10$

[dJ96] A. J. de Jong, Smoothness, semi-stability and alterations, Inst. Hautes Études Sci. Publ. Math. 83 (1996), no. 1, 51-93, http://www.numdam.org/item?id=PMIHES_1996__83__51_0. 6

[GM87] H. Gillet and W. Messing, Cycle classes and Riemann-Roch for crystalline cohomology, Duke Math. J. 55 (1987), no. 3, 501-538, https://doi.org/10.1215/S0012-7094-87-05527-x. $\uparrow 5,7$

[Kat89] K. Kato, Logarithmic structures of Fontaine-Illusie, Algebraic Analysis, Geometry and Number Theory (1989), 191-224. $\uparrow 7$

[Ked00] K. S. Kedlaya, Descent theorems for overconvergent F-crystals, Ph.D. thesis, Massachusetts Institute of Technology, 2000. $\uparrow 5$

[Ked04a] — Full faithfulness for overconvergent F-isocrystals, Geometric aspects of Dwork theory. Vol. I, II, Walter de Gruyter, Berlin, 2004, pp. 819-835. $\uparrow 5$

[Ked04b] —, A p-adic local monodromy theorem, Ann. of Math. (2) 160 (2004), no. 1, 93-184, https://doi.org/10.4007/annals.2004.160.93. $\uparrow 5$

[Keu16] J. Keum, Orders of automorphisms of K3 surfaces, Adv. Math. 303 (2016), 39-87, https://doi.org/10.1016/j.aim.2016.08.014. $\uparrow 9$

[Kul77] V. S. Kulikov, Degenerations of K3 surfaces and Enriques surfaces, Izv. Akad. Nauk SSSR Ser. Mat. 41 (1977), no. 5, 1008-1042, 1199. $\uparrow 1$

[LM18] C. Liedtke and Y. Matsumoto, Good reduction of K3 surfaces, Compos. Math. 154 (2018), no. 1, 1-35, https://doi.org/10.1112/S0010437X17007400. $\uparrow 2,4,6,8,9,10,13$

[LP16] C. Lazda and A. Pál, Rigid Cohomology over Laurent Series Fields, Algebra and Applications, vol. 21, Springer, 2016, http://dx.doi.org/10.1007/978-3-319-30951-4. 个3,6

[Mat95] S. Matsuda, Local indices of p-adic differential operators corresponding to Artin-Schreier-Witt coverings, Duke Math. J. 77 (1995), no. 3, 607-625, https://doi.org/10.1215/S0012-7094-95-07719-9. 9

[Mat15] Y. Matsumoto, Good reduction criteria for K3 surfaces, Math. Z. 279 (2015), no. 1-2, 241-266, http://dx.doi.org/10.1007/s00209-014-1365-8. $\uparrow 2,9$

[Mau14] D. Maulik, Supersingular K3 surfaces for large primes, Duke Mathematical Journal 163 (2014), no. 13, 2357-2425. $\uparrow 2$

[Ogu79] A. Ogus, Supersingular K3 crystals, Journées de Géométrie Algébrique de Rennes (Rennes, 1978), Vol. II, Astérisque, vol. 64, Soc. Math. France, Paris, 1979, pp. 3-86. $\uparrow 9$

[PP81] U. Persson and H. Pinkham, Degeneration of surfaces with trivial canonical bundle, Ann. of Math. (2) 113 (1981), no. 1, 45-66, http://dx.doi.org/10.2307/1971133. $\uparrow 1$

[RŠ76] A. N. Rudakov and I. R. Šafarevič, Inseparable morphisms of algebraic surfaces, Izv. Akad. Nauk SSSR Ser. Mat. 40 (1976), no. 6, 1269-1307. $\uparrow 12$

[SGA7-I] Groupes de monodromie en géométrie algébrique. I, Lecture Notes in Mathematics, Vol. 288, SpringerVerlag, Berlin-New York, 1972, Séminaire de Géométrie Algébrique du Bois-Marie 1967-1969 (SGA 7 I), Dirigé par A. Grothendieck. Avec la collaboration de M. Raynaud et D. S. Rim. $\uparrow 2$

[ST68] J.-P. Serre and J. Tate, Good reduction of abelian varieties, Ann. of Math. (2) 88 (1968), 492-517, http://dx.doi.org/10.2307/1970722. $\uparrow 2$

[Tsu99] T. Tsuji, Poincaré duality for logarithmic crystalline cohomology, Compositio Math. 118 (1999), no. 1, 11-41, https://doi.org/10.1023/A:1001020809306. $\uparrow 6$ 
(Chiarellotto) Dipartimento di Matematica "Tullio Levi-Civita", Università degli Studi di Padova, Via Trieste 63, 35121 Padova, Italy

E-mail address: chiarbru@math.unipd.it

(Lazda) Universiteit van Amsterdam, Korteweg-De Vries Institute for mathematics, P.O. Box 94248, 1090 GE, Amsterdam, the Netherlands

E-mail address: c.d.lazda@uva.nl

(Liedtke) TU München, Zentrum Mathematik - M11, Boltzmannstr. 3, 85748 Garching bei München, Germany

E-mail address: liedtke@ma.tum.de 